\title{
REZENSIONEN
}

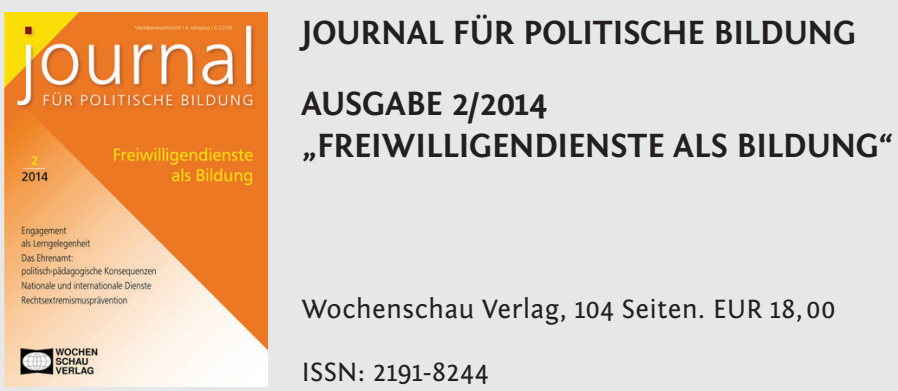

Schwerpunkt der Ausgabe 2/2014 der Vierteljahreszeitschrift „Journal für politische Bildung“ ist das Thema „Freiwilligendienste als Bildung“. Vier Aufsätze aus wissenschaftlicher, rechtlicher und praktischer Sicht betrachten die Freiwilligendienste aus unterschiedlichen Perspektiven. Es würde den Rahmen dieser Rezension sprengen, diese vier Beiträge in ihrer vollen inhaltlichen Breite zu würdigen. Stattdessen werden einzelne Aspekte heraus gegriffen, die dem Verfasser aus seiner eigenen Erfahrung mit den Freiwilligendiensten interessant erscheinen.

Prof. Dr. Thomas Klie, Rechtswissenschaftler und Leiter des Zentrums für zivilgesellschaftliche Entwicklung in Freiburg/Br., beschreibt die rechtlichen und politischen Rahmenbedingungen der verschiedenen FreiwilligendienstFormate und erinnert an die bereits im Koalitionsvertrag der CDU/CSU- und FDP-geführten Bundesregierung und in einem früheren Vorstoß des Bundesrats geäußerte Absicht, ein Freiwilligen-Statusgesetz zu verabschieden. ${ }^{1}$

Angesichts der Vielgestaltigkeit der Dienste (zurzeit gibt es in Deutschland sechs öffentlich geförderte und geregelte Formate) scheint dieser Hinweis - auch mit Blick auf die öffentliche Wahrnehmung - zunächst nicht ganz abwegig ${ }^{2}$. Ob dies in Form einer „Umbrella"-Regelung erfolgen soll, die eine rechtlich verbindliche Rahmenkonzeption für die bei allen Freiwilligendiensten auszumachenden Gemeinsamkeiten darstellt, oder als Artikel-Gesetz, in dem den einzelnen

1 Klie, Thomas, Freiwilligendienste, Rahmenbedingungen, politische und pädagogische Herausforderungen, in: Journal für politische Bildung, Ausgabe 2/2014, 8-16

2 Klie selbst spricht vom "Jugendfreiwilligendienstgesetz" anstatt die korrekte Bezeichnung ,Jugendfreiwilligendienstegesetz“ zu verwenden, die schon andeutet, dass es sich um mehrere Formate handelt. 
Freiwilligendienst-Formaten (FSJ, FÖJ, BFD etc.) jeweils einzelne Artikel gewidmet sind, diskutiert der Autor unter politischen und verfassungsrechtlichen Aspekten.

Als ein Ergebnis dieser Betrachtung ist Klies Plädoyer für eine einheitliche privatrechtliche Vertragsgestaltung anzusehen, die er ,gegenüber einer öffentlich-rechtlichen sowohl als traditionsgerecht als auch als inhaltlich angemessen“ (S. 12) bewertet. Die Bedeutung der rechtlichen Bestimmungen für die Qualitätskriterien der Freiwilligendienste stellt Klie mit Blick auf die Profilierung der Dienste als Bildungszeiten und im Zusammenhang mit der Frage der Arbeitsmarktneutralität heraus. Die in den vergangenen Jahren erfolgte Hervorhebung des Bildungscharakters von Freiwilligendiensten muss in den gesetzlichen Bestimmungen stärker als bisher berücksichtigt werden. Eine Forderung, der ebenso zuzustimmen ist wie der nach einer rechtlichen Regelung des Einsatzes der Freiwilligen als „arbeitsmarktneutral“ (S. 12).

Weiteren gesetzlichen Regelungsbedarf sieht Klie sowohl mit Blick auf Träger und Zentralstellen als auch hinsichtlich der staatlichen Aufgaben. Er möchte die „mittlere Steuerungsebene“ (Träger und Zentralstellen) gestärkt sehen und fordert eine klare Betonung ihrer Rechte und Pflichten, insbesondere in der Qualitätsentwicklung und der „Verschränkung mit regionalen Strukturen“" (S. 13).

Im Zusammenhang mit der Diskussion um staatliche Qualitätskriterien weist
Klie zu Recht darauf hin, dass sich das von Trägerseite eingeforderte Subsidiaritätsprinzip nicht auf die Wohlfahrtsverbände beschränkt, sondern für alle Träger gilt. In Übereinstimmung mit den Trägern sieht er die Aufgabe des Staates in der Rolle des Förderers und rechtlichen Rahmensetzers, ohne dabei den Bürokratieabbau zu vernachlässigen.

„Freiwilligendienste sind eine besondere Form des bürgerschaftlichen Engagements“ - so oder ähnlich heißt es in fast allen Darstellungen der Freiwilligendienste-Träger und der Bundesregierung. Ohne die Befürchtung einiger Seiten, bürgerschaftliches Engagement werde zurzeit von Regierungsseite nur noch unter dem Aspekt der Freiwilligendienste gesehen, direkt aufzugreifen, wirft Klie die Frage auf, ob an Stelle eines geforderten Freiwilligendienstrechts nicht die Forderung nach einem Recht der Engagementförderung zu treten habe. Er warnt vor der Vernachlässigung einer breit angelegten Engagementpolitik zu Gunsten einer Diskussion, die sich nur um Freiwilligendienste drehe. In der Frage der verfassungsrechtlichen Zuständigkeit (Bund oder Länder?), die eng mit der Frage der Finanzierung verknüpft ist, weist Klie auf die bestehenden unterschiedlichen, z. T. gegensätzlichen Sichtweisen und Interessenlagen hin. Er betrachtet Engagementförderung als Gemeinschaftsaufgabe, wobei das erwähnte Freiwilligen-Statusgesetz ein Puzzleteil sein könnte. Zur übergreifenden Regelung der von ihm aufgeworfenen Fragen regt Klie schließlich ein 
Engagement-Gesetzbuch an, in dem sowohl die Kompetenzen von Bund, Ländern und Kommunen als auch das Verhältnis von zivilgesellschaftlichen $\mathrm{zu}$ staatlichen Institutionen - und damit auch Rechte und Pflichten der Finanzausstattung der Freiwilligendienste geklärt werden könnten.

Der Artikel „Freiwilligendienste - Rahmenbedingungen, politische und pädagogische Herausforderungen" von Thomas Klie gibt den aktuellen Diskussionsstand und die zentralen rechtlichen und politischen Fragen der Freiwilligendienste übersichtlich und gut zusammengefasst wieder. Dass das Schwergewicht auf der rechtlichen und politischen Erörterung liegt, tut dem Beitrag - trotz der Ankündigung „pädagogischer Herausforderungen“ - keinen Abbruch.

Im zweiten Aufsatz mit dem Titel „Bildung in den Freiwilligendiensten" beschäftigen sich Prof. Dr. Thomas Olk (Professor für Sozialpädagogik und Sozialpolitik an der Martin-LutherUniversität Halle-Wittenberg und Vorsitzender des Sprecherrats des Bundesnetzwerks Bürgerschaftliches Engagement/BBE) und PD Dr. Ansgar Klein (Geschäftsführer des BBE) mit der Frage von Lernen und Bildung in den Freiwilligendiensten und ihrem Verhältnis zueinander. ${ }^{3}$ Auch wenn gleich zu Beginn des Artikels Freiwilligendienste als „Lerndienst" bezeichnet werden (S. 18), so bildet sich doch im weiteren

3 Olk, Thomas/Klein, Ansgar, Bildung in den Freiwilligendiensten, in: Journal für politische Bildung, Ausgabe 2/2014, 18-25
Verlauf ihrer Überlegungen eine differenzierte Betrachtungsweise für das heraus, was sich die Teilnehmenden an einem Freiwilligendienst im Laufe ihrer Tätigkeit aneignen.

Freiwilligendienste beinhalten auch neben ihrer Funktion als Teil des bürgerschaftlichen Engagements - Bildung, die den Freiwilligen durch Formen non-formalen und informellen Lernens nahe gebracht wird. In einem Lerndienst, mit dem in erster Linie die Vermittlung und Aneignung instrumentellen (und manchmal auch schulischen) Wissens suggeriert wird und der einer bloßen Zweckorientierung der Freiwilligendienste Vorschub leisten könnte, wird den Teilnehmenden das vermittelt, was sie für ihre Tätigkeit in der Einsatzstelle benötigen. ${ }^{4}$ Dem gegenüber verfolgen Freiwilligendienste ein mehrdimensionales Bildungskonzept, wie es im 12. Kinder- und Jugendbericht 2005 entwickelt wurde. Olk/Klein zitieren daraus: „Bildung wird als ein aktiver Prozess begriffen, in dem sich das Subjekt eigenständig und selbsttätig in der Auseinandersetzung mit der sozialen, kulturellen und natürlichen Umwelt bildet" ${ }^{\text {"5 }}$. Und weiter heißt es: „[...] der Erwerb kultureller, instrumenteller, sozialer und personaler Kompetenzen im Sinne einer

$4 \quad$ Lernen wird hier verstanden als Aneignung von Fakten, Verhaltensweisen und Tätigkeitsverläufen. Damit der kognitive Erwerb dieser Fakten, Verhaltensweisen und Tätigkeitsverläufe tatsächlich gelingt und nicht auf der Stufe des einmal Gehörten oder Erlebten stehen bleibt, werden verschiedene Lernformen (formal, nonformal oder informell) praktiziert.

5 BMFSFJ (Hg.), Zwölfter Kinder- und Jugendbericht, Berlin 2005, 83, zit. nach: Olk/Klein 2014, 20 
umfassenden Bildung des Subjekts [findet] nicht ausschließlich in der Schule, sondern in einer Vielzahl von Bildungsorten und Lernwelten statt.“ (ebd.) Die Unterscheidung zwischen Lerndienst und Bildungszeit sollte in der fachlichen Diskussion um die Funktion der Freiwilligendienste, ihre Möglichkeiten und Grenzen, in Zukunft beachtet werden - auch um der Gefahr ihrer Reduzierung auf billige Arbeitskräfte und einer Vernachlässigung ihres Bildungscharakters entgegen zu wirken.

Im weiteren Verlauf ihres Beitrags verweisen Olk/Klein auf Gisela Jakob, die den „Doppelcharakter von Bildungsjahr und Orientierungsphase einerseits sowie Übernahme sozialer Verantwortung und gemeinwohlorientiertem Handeln andererseits" in den Freiwilligendiensten hervorhebt und die Gleichzeitigkeit von Bildungserfahrungen und Tätigkeiten mit „Ernstcharakter" betont. ${ }^{6}$ Die vorliegenden Evaluationen bestätigen nach Olk/ Klein, dass die Erwartungen der Freiwilligen den Angeboten weitgehend entsprechen und die Befragten feststellen, dass der Freiwilligendienst zu ihrer persönlichen Entwicklung und zur Förderung ihrer Selbstständigkeit beigetragen habe.

Wenn die Autoren den Bundesfreiwilligendienst (BFD) ebenfalls als Lerndienst bezeichnen (S. $21 \mathrm{ff}$ ), so gelten dennoch auch für dieses Format die zuvor angestellten Überlegungen. Wenn

6 Jakob, G.: Freiwilligendienste zwischen Tradition und Erneuerung, 2004, $22 \mathrm{ff}$, nach: Olk/Klein 2014, 20 der BFD für über 27-Jährige als Teil des Konzepts des "lebenslangen Lernens“ verstanden werden sollte, so sei an dieser Stelle an die Reaktion von André Gorz, Ivan Illich und anderen erinnert, die dieser Idee bei ihrem erstmaligen Auftreten in den 1970er Jahren sofort kritisch gegenüber standen, weil sie eine Funktionalisierung und Instrumentalisierung des Lernens befürchteten. Genau diese Befürchtung greift aber auch im Zusammenhang mit dem BFD, mit welchem viele Freiwillige die Hoffnung auf einen Wiedereinstieg in den ersten Arbeitsmarkt verbinden. Infolgedessen werden sie bemüht sein, ihre Aufgaben fachlich optimal zu erledigen.

Was aber darüber hinausgeht und z.B. Zusammenhänge zwischen ihrer persönlichen Situation und gesellschaftlichen Entwicklungen verdeutlichen könnte, bleibt für sie scheinbar von nachrangiger Bedeutung. Von „Lernen" mag dann zwar noch die Rede sein, mitnichten aber von „Bildung“, wobei dabei völlig unerheblich ist, ob dieses Lernen formal, informell oder non-formal erfolgt.

Um dem entgegen zu treten, plädieren Olk/Klein für eine Stärkung der Lebensbewältigungs- und Lebensführungskompetenzen im BFD, eine Abgrenzung des BFD gegenüber arbeitsmarktpolitischen Maßnahmen und gegen eine Vernachlässigung des Bildungscharakters des BFD, um die „Dachmarke“ Freiwilligendienste nicht zu beschädigen.

Angesichts der Tatsache, dass im BFD insbesondere in den östlichen 
Bundesländern überwiegend Freiwillige aktiv sind, die älter als 27 Jahre sind, und viele dieser Personen nach Aussagen der pädagogischen Mitarbeiter/innen keine große Begeisterung zeigen, wenn sie mit dem Bildungsaspekt in den Freiwilligendiensten konfrontiert werden, stellt sich hier das Problem der Bildung im Freiwilligendienst noch einmal unter einem anderen, für die Träger neuen Blickwinkel.

Unter Berücksichtigung der besonderen Herausforderungen, die sich bei der Entwicklung eines geeigneten Bildungskonzepts für diese Gruppe stellen, fordern die Autoren, das Bildungssubjekt und die Bildungsbiografie in den Mittelpunkt zu stellen und heben in diesem Zusammenhang nochmals das non-formale und informelle Lernen hervor, das in systematische Reflexionsprozesse überführt werden müsse (S. 23). Nicht unkritisch ist jedoch ihr Hinweis auf Zertifizierungsformen und den Europäischen Qualifikationsrahmen (EQR) zu sehen, um dem informellen Kompetenzerwerb eine Anerkennung vergleichbar den Bildungsabschlüssen $\mathrm{zu}$ verschaffen (S. 23). Die damit verbundene Gefahr einer „Formalisierung des Informellen“ und damit das Ende des informellen Lernens ist nicht von der Hand zu weisen. Obwohl die Verwendung der Bezeichnung „Lerndienst“ für einen Freiwilligendienst hier abgelehnt wird, ist es der Verdienst der beiden Autoren, sich mit den Lernformen in den Freiwilligendiensten und ihrem Bildungscharakter gründlich auseinander gesetzt zu haben. Insbesondere die Erörterung dieser Aspekte im BFD verdient Anerkennung, da sie in Wissenschaft und Praxis erst langsam Eingang finden. Das Problem der Nachbetreuung über 27-jähriger BFD’ler/innen harrt allerdings weiterhin einer Lösung, für die jedoch nicht die Wissenschaft, sondern Einsatzstellen und Träger verantwortlich sind.

Vier Mitarbeiter eines Bildungszentrums des Bundes (Clemens Antoni, Michael König, Hubert Kolling, Manfred Pappenberger) widmen sich im dritten Beitrag der pädagogischen Begleitung im Bundesfreiwilligendienst. ${ }^{7}$ Unter Bezugnahme auf die „Rahmenrichtlinie für die pädagogische Begleitung im Bundesfreiwilligendienst" und die „Pädagogische Rahmenkonzeption zur Seminararbeit an den staatlichen Bildungszentren“ werden zunächst das Lernverständnis und die didaktischen Prinzipien dargestellt.

Die Autoren verstehen die Heterogenität der Teilnehmerschaft an den BFDSeminaren als Bereicherung, weil sie die gesellschaftliche Realität abbilde. Über drei Module sollen die Freiwilligen vom Ich (im Einstiegsseminar) über das $D u$ (im Modul 2 über soziale Kompetenzen) zum Wir (im Modul 3 zur politischen Bildung) gelangen. Basierend auf den Erkenntnissen der Lernpsychologie wird der Schwerpunkt des Lernens in praktischer Betätigung (in Projekten in den Einsatzstellen) gesehen. Einer kurzen Schilderung von Einstiegs- und

\footnotetext{
7 Antoni, Clemens u.a., Pädagogische Begleitung im Bundesfreiwilligendienst, in: Journal für politische Bildung, Ausgabe 2/2014, 26-35
} 
Kompetenzseminar folgt die ausführliche Darstellung der politischen Bildung, die in Form eines fünftägigen Seminars an einem Bildungszentrum des Bundes gemäß BFD-Gesetz für alle BFD'ler/innen obligatorisch ist. Warum sind nach Meinung der Autoren die Bildungszentren des Bundes besonders geeignet, politische Bildung zu vermitteln? Diese Frage soll sich im Anschluss an eine Aufzählung zentraler Aspekte der politischen Bildung unter Berufung auf den sogenannten Beutelsbacher Konsens beantworten, demzufolge in der außerschulischen politischen Bildung eine „von den weltanschaulichen Hintergründen der Träger unabhängige fachliche Professionalität" (S. 30) vorherrschen soll. Die nachvollziehbare Forderung nach fachlicher Professionalität wird mit einer Unterstellung verknüpft: Freie Träger versuchen (nur) ihre weltanschaulichen und politischen Positionen einzubringen, die Teilnehmenden also quasi zu missionieren. Damit wird den Freien Trägern a priori ein Verstoß gegen das Indoktrinationsverbot und ein weltanschaulicher Alleinvertretungsanspruch unterstellt; bei gleichzeitiger Annahme, die staatlichen Bildungszentren seien dagegen gefeit und per se objektiv oder neutral. Bleibt die Frage, ob die dort Lehrenden keine politischen Positionen haben oder diese immer so weit zurücknehmen können, dass ihre Position nicht durchscheint. Unterschiedliche oder auch gegensätzliche Anschauungen, wie im Gebot der Kontroversität gefordert, kommen bei Freien Trägern ebenso gut zur Geltung wie an den Bildungszentren des
Bundes. Selbst wer der Fiktion anhängt, der Staat sei neutral, wird zugestehen müssen, dass die ihn repräsentierenden Personen Subjekte mit eigenen Einstellungen und Interessen sind und die Institution „Staat" selbst bisweilen auch ideologisch gesteuert ist. ${ }^{8}$

Zur Lösung des Problems, wie in pädagogischen Zusammenhängen, die sich neutral verhalten wollen, mit weltanschaulichen Orientierungen umzugehen ist, sei hier an die Position Max Webers erinnert, die er im Werturteilsstreit der deutschen Soziologie vertreten hat: Werte sind nicht wissenschaftlich beweisbar, sondern beruhen auf Interessen, Einstellungen, Traditionen etc. Es ist ein Gebot der wissenschaftlichen (und pädagogischen) Redlichkeit, die eigenen Werte oder Grundannahmen dem Publikum transparent zu machen und Alternativen aufzuzeigen, damit dieses die Möglichkeit hat, eine eigene Position zu entwickeln und nicht nur eine Auffassung als die allein richtige kennen lernt. Nur über Transparenz und Pluralität ist also eine Annäherung an Neutralität möglich. Fachliche Professionalität, die durch die weltanschauliche Orientierung angeblich

8 So lautet ein Argument in dem von Mitarbeitern des BMFSFJ und des BAFZA veröffentlichten Kommentar zum BFDG gegen die Einführung des Trägerprinzips im BFD, ,dass es dem Bund, (...), auch aus ideologischen Gründen nicht möglich gewesen wäre, die aus dem Zivildienst zu erhaltenden Strukturen in die vorhandenen Strukturen der Jugendfreiwilligendienste zu übertragen." (Hübner/Mansfeld, Bundesfreiwilligendienstgesetz, München 2014, 14, Rn. 52) (Hervorhebungen so im Original). Das Buch ist ebenfalls in dieser Voluntaris-Ausgabe rezensiert. 
gefährdet ist, hängt jedoch vielmehr von Personen, Konzeptionen und Methoden $\mathrm{ab}$ als von scheinbar wertneutral arbeitenden Institutionen. Und wie verhält sich die Befürchtung einer gefährdeten Professionalität in der politischen Bildung gegenüber Freien Trägern, die sich als überkonfessionell und weltanschaulich neutral betrachten wie z. B. dem Deutschen Roten Kreuz, dem Paritätischen Gesamtverband oder dem Internationalen Bund?

Einer solchen Befürchtung steht außerdem die Erfahrung aus der Praxis entgegen, dass die jeweilige weltanschauliche Ausrichtung der Träger für die Entscheidung der Freiwilligen nur eine nachgeordnete Rolle spielt. Vorrangiges Kriterium für die Trägerwahl der Freiwilligen ist die Art der Einsatzstelle, in der sie ja bekanntlich auch den Großteil ihrer Zeit während des Dienstes verbringen. Das wiederum sollte jedoch den Freien Trägern zu denken geben, die ihre Raison d'être auch aus ihrer weltanschaulichen Unterschiedlichkeit beziehen. ${ }^{9}$ Wenn die Autoren unter Berufung auf §4.4 BFDG schreiben, dass „die Behandlung politischer Fragen auch nicht auf die Darstellung einer einseitigen Meinung beschränkt oder BFD'ler [...] nicht zugunsten oder zuungunsten einer bestimmten politischen Meinung beeinflusst werden

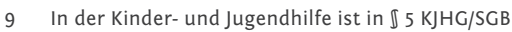
VIII das Wunsch- und Wahlrecht von Eltern und Kindern festgelegt. Um auch in den Freiwilligendiensten weltanschauliche Pluralität zu gewährleisten, hat der Gesetzgeber öffentlichen und Freien Trägern die Möglichkeit eröffnet, als Anbieter von Freiwilligendiensten tätig zu sein. (dürfen)“ (S. 30), so sollte dies in einer sich pluralistisch verstehenden Gesellschaft selbstverständlich sein.

Trotz der hier angeführten kritischen Aspekte, insbesondere dem Alleinvertretungsanspruch in der politischen Bildung, soll nicht bestritten werden, dass die pädagogische Begleitung im BFD an den Bildungszentren des Bundes, wie sie in dem Artikel beschrieben wird, auf einem fachlich hohen Niveau geleistet wird, das den Freiwilligen Chancen für intellektuelle Bereicherungen und Erfahrungsaustausch bietet.

Im vierten und letzten Beitrag betrachtet Benjamin Haas, Mitherausgeber von Voluntaris - Zeitschrift für Freiwilligendienste und früherer Mitarbeiter der „Koordinierungsstelle weltwärts“, die Internationalen Freiwilligendienste als "global-politische Lernorte“. ${ }^{10}$ Nachdem zunächst die Verbindung von Globalem Lernen und politischer Bildung allgemein Thema ist, steht das weltwärts-Programm und seine Akzentuierung auf den Bildungsaspekt im Zentrum. Die anschließend präsentierten Erkenntnisse über den Zusammenhang von Globalem Lernen und politischer Bildung beruhen auf den Ergebnissen der Programmevaluation von weltwärts. Am Schluss geht der Verfasser noch auf die Bedeutung des sogenannten „Incomings“ ein. Mehr noch als andere, v. a. internationale, Freiwilligendienste muss sich weltwärts dem

10 Haas, Benjamin, Internationale Freiwilligendienste als global-politische Lernorte, in: Journal für politische Bildung, Ausgabe 2/2014, 36-44 
Anspruch verpflichtet fühlen, Globales Lernen in die politische Bildung einzubringen. War es doch die Kritik der OECD an Deutschland, zu wenig für die Entwicklung eines Wissens um entwicklungspolitische Zusammenhänge getan zu haben, die als ein Auslöser für dieses Programm gesehen werden kann. ${ }^{11}$ Nach einer Erläuterung des Begriffs „Globales Lernen“ werden die damit verfolgten Ziele (1. Vermittlung von Verständnis der globalen Dimension, 2. Stärkung der Persönlichkeit, 3. Erwerb globaler Handlungskompetenz) vorgestellt und die Verbindung zur politischen Bildung beschrieben. Der Bezug zu weltwärts wird deutlich, wenn Haas schreibt: „Sie (die „weltwärts“-Freiwilligen, H. B.) sollen Kompetenzen der interkulturellen Kommunikation und sozialen Verantwortung erwerben und die Abhängigkeit des eigenen Lebens und Handelns in der globalisierten Welt erfahren, verstehen und reflektieren" (S. 38). Im nächsten Schritt geht der Autor auch auf die Kritik an der Methode des Globalen Lernens ein (Reproduktion von Stereotypen und überholten Kulturverständnissen) und fordert „eine fundierte macht- und rassismuskritische Auseinandersetzung mit den Methoden und Inhalten Globalen Lernens.“ (S. 39).

So sehr man diesen Wunsch auch teilen mag, muss man sich jedoch auch die Frage stellen, ob ein staatlich finanziertes Programm mit einer solchen Forderung nicht selbst überfordert ist. Ohne

11 OECD (Hg.): DAC Peer Review Germany 2006, $25 \mathrm{f}$ hier für einen vorauseilenden Gehorsam seitens der entwicklungspolitisch Aktiven zu plädieren, sollte man sich aber über die Interessen des „Globalen Nordens“ an Entwicklungszusammenarbeit keinen Illusionen hingeben. Gleichwohl können auch durch die Erfahrung des Scheiterns solcher Erwartungen Lerneffekte erzielt werden.

Auch weltwärts selbst hat einen Lernprozess durchlaufen: stand zu Beginn das Motto „Lernen durch Helfen“ im Vordergrund, so ist die überholte Hilfsperspektive zugunsten des Bildungsaspekts inzwischen in den Hintergrund getreten und hat dem Anspruch auf gegenseitiges Lernen Platz gemacht. Damit verbunden ist auch die von Beginn an seitens der Entsendeorganisationen aufgestellte Forderung nach einer „Süd-Nord-Komponente“ in weltwärts, durch die auch Freiwillige aus dem „Globalen Süden“ nach Deutschland kommen sollten. Erste Ansätze in diese Richtung sind seit Anfang 2014 zu erkennen, als seitens des Bundesministeriums für wirtschaftliche Zusammenarbeit und Entwicklung auch Mittel für Incoming bereitgestellt wurden. Verstärkt wird diese Möglichkeit auch durch die dafür geeignete Förderstruktur des Bundesfreiwilligendienstes.

In seinem Fazit betont Haas, dass die weltwärts-Freiwilligen „eine gesellschaftlich privilegierte Gruppe" seien und fordert, breitere gesellschaftliche Zielgruppen zu erreichen (S. 43). Mit diesem Problem sind alle Freiwilligendienst-Formate seit Langem konfrontiert und sicher haben die etablierten 
Strukturen und die Geschichte der Freiwilligendienste ihren Anteil am Status quo. Es muss aber auch die Frage erlaubt sein, ob die bisher nicht erreichten Zielgruppen nur aus diesen Gründen keinen Zugang finden konnten oder ob es nicht vielmehr andere Ursachen wie z.B. fehlendes Selbstbewusstsein, fehlende Sprachkenntnisse etc. sind, die junge Menschen mit formal niedrigem Bildungsabschluss davon abhalten, sich für einen Freiwilligendienst zu interessieren. Freiwilligendienste sind, wie der Name schon sagt, freiwillig! Niemand sollte dazu direkt oder indirekt gezwungen werden und Freiwilligendienste sind auch nicht der Schlüssel zur Lösung aller sozialen Probleme.

Insgesamt stellt der Artikel von Benjamin Haas eine lesenswerte Erörterung der Probleme globalen politischen Lernens dar, die zur weiteren Beschäftigung mit dem Thema anregt.

Bildung in den Freiwilligendiensten ist ein weites Feld, wie die Lektüre dieser vier Artikel einmal mehr belegt. Es bleibt zu hoffen, dass dieses Thema auch über den Kreis der Fachöffentlichkeit hinaus Beachtung finden wird - ohne sie auf die instrumentellen Aspekte von Zertifizierung oder „Förderung der Bildungs- und Beschäftigungsfähigkeit“ zu reduzieren. Stattdessen sollte auch in der Bildung dem Eigen-Sinn der Freiwilligendienste Rechnung getragen werden.

\section{Hartmut Brombach}

Referatsleiter Freiwilligendienste in der Zentralen Geschäftsführung des Internationalen Bundes (IB) ${ }^{12}$

hartmut.brombach@internationaler-bund.de

12 Die in dem Beitrag zum Ausdruck gebrachten Positionen stellen die persönlichen Auffassungen des Autors dar und nicht die offiziellen Positionen des IB. 